\title{
Hochschild cohomology of a generalisation of canonical algebras
}

\author{
María Andrea Gatica and Marcelo Lanzilotta
}

\begin{abstract}
We compute the Hochschild cohomology groups of a family of finite dimensional algebras, the toupie algebras, characterized by having the same associated ordinary quiver as canonical algebras and any admissible ideal. Moreover, we determine the simple connectedness of them and we give necessary and sufficient conditions for rigidity.
\end{abstract}

\section{Introduction}

Let $A$ be a finite dimensional indecomposable algebra (associative with unit) over an algebraically closed field $k$. We assume that the algebra $A$ is basic, that is, $A=k Q / I$ for some finite quiver $Q$ and an admissible ideal $I$ of the path algebra $k Q([\mathrm{ARS}])$.

The Hochschild cohomology groups $H^{i}(A, X)$ of $A$ with coefficients in an $A$-bimodule $X$ were defined by Hochschild [Ho]. When $X=A$ we shall denote $H^{i}(A)=H^{i}(A, A)$ the i-th Hochschild cohomology group of $A$. In particular, the vanishing of the low dimensional groups $H^{i}(A), 1 \leq i \leq 3$, has classical interpretations and plays an important role in the representation theory of algebras: $H^{1}(A)$ has a close relation with the simple connectedness of $A$ (see [AP], [H1]); $H^{2}(A)$ and $H^{3}(A)$ are related to the rigidity properties of $A$ (see [G]).

2000 Mathematics Subject Classification. Mathematics Subject Classification : $16 \mathrm{E} 40$

Key words: Cohomology, Hochschild, finite dimensional algebra, canonical algebra. 
In general it is not easy to compute the Hochschild cohomology groups of a given algebra. Direct formulas exist in some particular cases (see [H1], [C2], [L], [BLM], [GR1], [GR2], [GRey]) and some exact sequences of cohomology groups have been obtained (see [H1], [C3], [MiP]). Recently, Bustamante, Dionne and Smith have proved that for some particular families of algebras there is a Mayer-Vietoris sequence of Hochschild cohomology groups ([BDS]).

An algebra $A=k Q / I$ is called a toupie algebra if $Q$ is a toupie quiver, that is, $Q$ has a unique source and a unique sink, and, for any other vertex $x$ there is exactly one arrow having $x$ as source and exactly one arrow having $x$ as target. The canonical algebras, introduced by $[R]$, are an example of such algebras. In fact, the toupie algebras were defined in [CDHL] as a generalisation of canonical algebras, because they have the same ordinary quiver and any admissible ideal. In this work, the authors determined when a toupie algebra is hereditary, tilted, quasi-tilted, shod, weakly shod or laura. The interest in such algebras lies in the fact that every ordinary quiver of a triangular algebra may be thought as a union (not necessarily disjoint) of toupie subquivers. Therefore it could be possible to determine some properties of families of algebras which result from gluing together several toupie algebras.

The main purpose of this paper is to compute the Hochschild cohomology groups of a toupie algebra. In particular, we determine the simple connectedness and we give necessary and sufficient conditions for rigidity.

This work consists of four sections. In section 2, we fix notations and briefly recall the definitions and results that will be needed throughout this paper. In section 3, we compute the Hochschild cohomology groups of toupie algebras. In the fourth and last section, we study their simple connectedness and rigidity, and we present some examples which arise from gluing several toupie algebras together in order to illustrate the use of the obtained result.

\section{Preliminaries}

\subsection{Path Algebras.}

A quiver $Q=\left(Q_{0}, Q_{1}\right)$ is a finite oriented graph where $Q_{0}$ is the set of vertices and $Q_{1}$ is the set of arrows. We denote by $s, t: Q_{1} \rightarrow$ 
$Q_{0}$ the maps associating to each arrow its starting and ending point respectively.

The path algebra $k Q$ is the $k$-vector space with basis all the paths in $Q$, including trivial paths $e_{x}$ of length zero, one for each vertex $x \in Q_{0}$. The multiplication of two elements of the basis is the concatenation of paths if they are composable, and zero otherwise.

A relation from $x$ to $y$ is a linear combination $\rho=\sum_{i=1}^{m} \lambda_{i} w_{i}$ such that, for each $1 \leq i \leq m, \lambda_{i}$ is a non-zero scalar and $w_{i}$ is a path of length at least two from $x$ to $y$. We say that $\rho$ is a monomial relation if $m=1$ (i.e. $\rho=w_{1}$ ). A set of monomial relations $\left\{w_{i}\right\}_{i=1, . ., u}$ is called an overlapping if the paths $w_{i}$ and $w_{i+1}$ share at least an arrow, for all $i=1, . ., u-1$.

The ideal $I$ generated in the path algebra $k Q$ by a set of relations is admissible if $I \subset J^{2}$ and $J^{m} \subset I$ for some $m \in \mathbb{N}$, where $J$ is the ideal generated by all arrows. The pair $(Q, I)$ is then called a bound quiver. It is well-known (see [ARS] for example) that for every basic finite dimensional algebra $A$ over an algebraically closed field $k$ there exists a surjective $k$-algebra morphism $v: k Q \rightarrow A$ with admissible kernel $I_{v}$, where $Q$ is the ordinary quiver of $A$. Thus we have $A \simeq k Q / I_{v}$. The bound quiver $\left(Q, I_{v}\right)$ is called a presentation of $A$.

Let $A=k Q / I$ be an algebra. A vertex $i$ in $Q$ is called a sink if there is no arrow in $Q$ starting at $i$ and a source if there is no arrow ending at $i$.

It is well-known that if $A=k Q / I$, then the category $A-\bmod$ of finitely generated left $A$-modules is equivalent to the category of all bound (finite-dimensional) representations of $(Q, I)$. Therefore we may identify a module $M$ with the corresponding representation $(M(x), M(\alpha))_{x \in Q_{0}, \alpha \in Q_{1}}$. For each $x \in Q_{0}$, we denote by $S_{x}$ the corresponding simple $A$-module associated to $x$ and $P_{x}, I_{x}$ will denote the projective cover and injective envelope of $S_{x}$, respectively. Then it is easy to see that there is a vector space isomorphism between $\operatorname{Hom}_{A}\left(P_{x}, M\right)$ and $M(x)$.

We say that an algebra $B$ is a convex subcategory of $A=k Q / I$ if there is a path closed full subquiver $Q^{\prime}$ of $Q$ such that $B=k Q^{\prime} /(I \cap$ $\left.k Q^{\prime}\right)$. This means that any path in $Q$ with starting and ending point in $Q^{\prime}$ lies entirely in $Q^{\prime}$.

The following well-known fact will be necessary in the sequel. 
Lemma 2.1. Let $B$ be a convex subcategory of $A$ and let $X, Y$ be elements of $B-\bmod$. There is an isomorphism between $\operatorname{Ext}_{A}^{i}(X, Y)$ and $\operatorname{Ext}_{B}^{i}(X, Y)$, for all $i \neq 0$.

We refer to $[\mathrm{ARS}]$ for more details.

\subsection{Toupie Algebras.}

A quiver $Q$ is called toupie if it has a unique source 0 and a unique sink $\omega$, and, for any other vertex $x$ there is exactly one arrow having $x$ as source and exactly one arrow having $x$ as target:

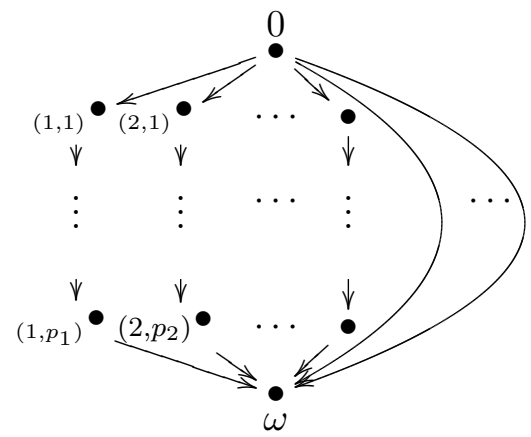

The distinct paths from 0 to $\omega$ are the branches of $Q$. An overlapping $\left\{w_{i}\right\}_{i=1, \ldots, u}$ on a branch is said to be a full overlapping if $w_{1}$ starts at 0 and $w_{u}$ ends at $\omega$. Given an overlapping $\left\{w_{i}\right\}_{i=1, \ldots, u}$ we construct a new sequence of relations $\left\{\omega_{i}\right\}$ in the following way: $\omega_{1}=w_{1}$, and $\omega_{2}=w_{2}$, and define recursively $\omega_{i}=w_{j}$ with $j$ minimum such that $t\left(\omega_{i-2}\right) \leq s\left(w_{j}\right)<t\left(\omega_{i-1}\right)$. Is easy to observe that the sequence $\left\{\omega_{i}\right\}_{i=1, \ldots, \nu}$ is also an overlapping. This sequence is called a real overlapping. We associate to each full overlapping $\left\{w_{i}\right\}_{i=1, \ldots, u}$ its real overlapping sequence $\left\{\omega_{i}\right\}_{i=1, \ldots, \nu}$. If $t\left(\omega_{\nu}\right)=\omega$ then we get a real full overlapping.

We say that $A$ is a toupie algebra if $A=k Q / I$ with $Q$ a toupie quiver, $k$ an algebraically closed field and $I$ any admissible ideal.

The following notation will be useful in the next section. Given a toupie algebra we denote by:

- $d=\operatorname{dim}_{\mathrm{k}} e_{\omega} A e_{0}$

- $b_{1}, \cdots, b_{B}$ be the branches. Then: 
$\diamond b_{1}, b_{2}, \ldots, b_{b}$ are of length greater than or equal to two and contain no monomial relations (notice that these branches could be related between them);

$\diamond b_{b+1}, \ldots, b_{b+m}$ contain monomial relations starting at 0 without full overlapping;

$\diamond b_{b+m+1}, \ldots, b_{b+m+f}$ contain full overlappings;

$\diamond b_{b+m+f+1}, \ldots, b_{b+m+f+o}$ contain at least one monomial relation and contain no relation starting at 0 ;

$\diamond b_{b+m+f+o+1}, \ldots, b_{b+m+f+o+a}$ are of length one;

- For every natural number $l$ we define the number $f(l)$ in the following way.

For every full overlapping on a branch, we construct its real overlapping. Then, in case that its real overlapping is full (that is, it is a real full overlapping), we count the number of relations that take part in it. Hence, for all natural number $l$, $f(l)=$ number of real full overlappings branches with $l$ relations.

\subsection{Hochschild cohomology.}

We recall the definition of the Hochschild cohomology groups $H^{i}(A)$ of an algebra $A$. Consider the $A$-bimodule $A$ and the complex $C^{\bullet}=$ $\left(C^{i}, d^{i}\right)$ defined by:

- $C^{i}=0, d^{i}=0$ for $i<0$,

- $C^{0}=A, C^{i}=\operatorname{Hom}_{k}\left(A^{\otimes i}, A\right)$ for $i>0$, where $A^{\otimes i}$ denotes the $i$-fold tensor product $A \otimes_{k} \cdots \otimes_{k} A, d^{0}: A \rightarrow \operatorname{Hom}_{k}(A, A)$ the map $d^{0}(x)(a)=a x-x a$ and

- $d^{i}: C^{i} \rightarrow C^{i+1}$ defined by

$$
\begin{aligned}
\left(d^{i} f\right)\left(a_{1} \otimes \cdots \otimes a_{i+1}\right) & =a_{1} f\left(a_{2} \otimes \cdots \otimes a_{i+1}\right) \\
& +\sum_{j=1}^{i}(-1)^{j} f\left(a_{1} \otimes \cdots \otimes a_{j} a_{j+1} \otimes \cdots \otimes a_{i+1}\right) \\
& +(-1)^{i+1} f\left(a_{1} \otimes \cdots \otimes a_{i}\right) a_{i+1},
\end{aligned}
$$

for $f \in C^{i}$ and $a_{1}, \ldots, a_{i+1} \in A$.

Then $H^{i}(A)=H^{i}\left(C^{\bullet}\right)=\operatorname{Ker} d^{i} / \operatorname{Im} d^{i-1}$ is the $i$-th Hochschild cohomology group of $A$ with coefficients in $A$, see [Ho]. Moreover, $H^{i}(A)$ is a $k$-vector space. 
The Hochschild cohomology groups of a given algebra are generally hard to compute by using the definition. For this reason, one often tries to find alternative methods for computing these groups. For example, we can use an inductive method to compute the Hochschild cohomology groups when we are dealing with triangular algebras.

In fact, if $A=k Q_{A} / I$ is a triangular algebra then the associated quiver has at least one sink and one source, and this allows us to describe $A$ as a one point extension algebra in the following way.

If $x$ is a source in $Q_{A}$ then the full convex subcategory $A_{x}$ of $A$ consisting of all objects except $x$, has $Q_{A_{x}}$ as the quiver obtained from $Q_{A}$ by deleting $x$ and all arrows starting at $x$. Any presentation $\left(Q_{A}, I\right)$ of $A$ yields (by restriction) an induced presentation $\left(Q_{A_{x}}, I^{\prime}\right)$ of $A_{x}$.

The $A$-module $M=\operatorname{rad} P_{x}$ has a canonical $A_{x}$-module structure, and $A$ is isomorphic to the one point extension algebra

$$
A_{x}[M]=\left(\begin{array}{ll}
k & 0 \\
M & A_{x}
\end{array}\right)
$$

where the operations are the usual addition of matrices and the multiplication induced by the $A_{x}$-module structure of $M$.

The next theorem, due to Happel [H1], is useful for computing the Hochschild cohomology groups of the algebras considered in this article.

Theorem 2.2. [H1] Let $A=A_{x}[M]$ be a one point extension of $A_{x}$ by an $A_{x}-$ module $M$. Then there exists a long exact sequence of $k$-vector spaces connecting the Hochschild cohomology of $A$ and $A_{x}$ :

$$
\begin{gathered}
0 \rightarrow H^{0}(A) \rightarrow H^{0}\left(A_{x}\right) \oplus k \rightarrow \operatorname{Hom}_{A_{x}}(M, M) \rightarrow H^{1}(A) \rightarrow H^{1}\left(A_{x}\right) \rightarrow \\
\operatorname{Ext}_{A_{x}}^{1}(M, M) \rightarrow \ldots \rightarrow H^{i}(A) \rightarrow H^{i}\left(A_{x}\right) \rightarrow \operatorname{Ext}_{A_{x}}^{i}(M, M) \rightarrow H^{i+1}(A) \rightarrow \ldots
\end{gathered}
$$

Observe that this result is very useful when most terms in the sequence vanish. In particular, we get the following consequence.

Corollary 2.3. Let $A=A_{x}[M]$, with $A_{x}$ an indecomposable triangular algebra. If $H^{i}\left(A_{x}\right)=0$ for all $i \geq 1$, then 


$$
H^{i}(A)= \begin{cases}k & \text { if } i=0, \\ k^{\operatorname{dim}_{\mathrm{k}} \operatorname{Hom}_{A_{x}}(M, M)-1} & \text { if } i=1, \\ \operatorname{Ext}_{A_{x}}^{i-1}(M, M) & \text { for all } i \geq 2 .\end{cases}
$$

Proof. Since $A_{x}$ is an indecomposable triangular algebra, $Z\left(A_{x}\right)=$ $Z(A)=k$ so, $H^{0}(A)=H^{0}\left(A_{x}\right)=k$ and our claim follows directly from Theorem 2.2.

Finally, we recall two results about Hochschild cohomology groups of algebras that will be necessary in the next section.

The first one, due to Cibils [C1], is related with the Hochschild cohomology groups of an algebra $A=k Q / I$ which is schurian (i.e. $\operatorname{dim}_{k} \operatorname{Hom}_{A}\left(P, P^{\prime}\right) \leq 1$, for any $P, P^{\prime}$, indecomposable projective $A$ modules) and semicommutative (i.e. if $w, w^{\prime}$ are parallel paths in $Q$ with $w \in I$, then $\left.w^{\prime} \in I\right)$.

Theorem 2.4. [C1] Let $A=k Q / I$ be a schurian and semicommutative algebra. Then $H^{0}(A)=k, \operatorname{dim}_{k} H^{1}(A)=1-\left|Q_{0}\right|+\left|Q_{1}\right|$ and $H^{i}(A)=0$, for all $i \geq 2$.

The second result, due to Green, Marcos and Snashall [GMS], is concerned with the Hochschild cohomology ring of a one point extension algebra.

Theorem 2.5. [GMS] Let $A=B[M]$ be a one point extension of $B$ by a $B$-module $M$. Then, the graded homomorphism associated to the Happel long exact sequence

$$
\delta^{*}: \operatorname{Ext}_{B}^{*}[M, M] \rightarrow H^{*}(A)[-1]
$$

has image in the annihilator of $\coprod_{n \geq 1} H^{n}(A)$, where $H^{*}(A)[-1]$ is the graded $Z$-module $X$, where $X_{n}=H^{n+1}(A)$.

\section{The results}

In this section we compute all the Hochschild cohomology groups of toupie algebras. In order to do this, we consider these algebras as one point extension algebras. In fact, if $A$ is a toupie algebra (see 
2.2) then $A=A_{0}[M]$ where $M=\operatorname{rad} P_{0}$ and $A_{0}$ is the full convex subcategory of $A$ consisting of all objects except 0 .

Then, since $A=A_{0}[M]$, we have that $A_{0}=k Q_{A_{0}} /\left(I \cap k Q_{A_{0}}\right)$ with $Q_{A_{0}}$ :

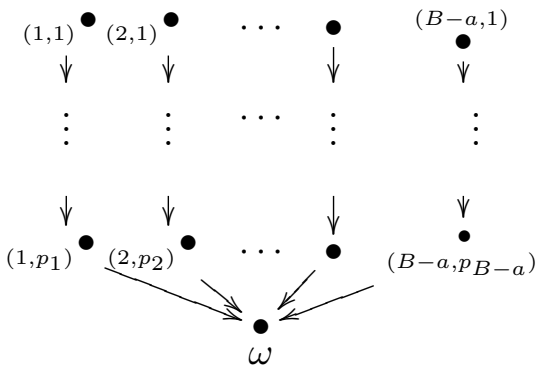

Bearing in mind that we will compute the Hochschild cohomology groups of this particular classes of algebras using the long exact sequence in Theorem 2.2, it will be necessary to compute the groups $H^{i}\left(A_{0}\right)$ and $\operatorname{Ext}_{A_{0}}^{i}(M, M), i \geq 0$.

Remark 3.1. Notice that $A_{0}$ is a semicommutative schurian algebra such that $1-\left|Q_{0}\right|+\left|Q_{1}\right|=1-\left(p_{1}+p_{2}+\cdots+p_{B-a}+1\right)+\left(p_{1}+p_{2}+\cdots+p_{B-a}\right)=0$. Then, it follows directly from Theorem 2.4 that

$$
H^{i}\left(A_{0}\right)= \begin{cases}k & \text { if } i=0, \\ 0 & \text { otherwise. }\end{cases}
$$

We recall that for a given $A$-module $M$, the support of $M$ is given by

$$
\operatorname{supp} M=\left\{x \in Q_{0}: M(x) \neq 0\right\} .
$$

Remark 3.2. Let $M_{1}, M_{2}$ be two A-modules such that $\operatorname{supp} \operatorname{top}\left(M_{1}\right) \cap$ $\operatorname{supp} M_{2}=\emptyset$. Then $\operatorname{Hom}_{A}\left(M_{1}, M_{2}\right)=0$.

We fix the last parameter: let $r$ be the number of indecomposable summands of $M$ with support contained in the set $\{(j, l)$, for all $1 \leq$ $\left.j \leq b, 1 \leq l \leq p_{j}\right\} \cup\{\omega\}$. 
Now we are able to prove the next lemma, that will be used to compute the Hochschild cohomology groups of a toupie algebra $A=$ $k Q / I$.

Lemma 3.3. Let $A_{0}=A /<e_{0}>$ and let $M=\operatorname{rad} P_{0}$. Then

$$
\operatorname{Ext}_{A_{0}}^{i}(M, M)= \begin{cases}k^{r+m+f+o+d . a} & \text { if } i=0, \\ k^{r-b+(b+a-d+f(1)) d} & \text { if } i=1, \\ k^{f(i) . d} & \text { if } i \geq 2,\end{cases}
$$

where $r, d, b, m, f, o, a, f(i)$ were defined previously.

Proof. It is easy to see that

$$
M=\operatorname{rad} P_{0}=M_{b} \oplus\left(\bigoplus_{j=b+1}^{b+m+f+o} M_{j}\right) \oplus P_{\omega}^{a}
$$

where:

- $M_{b}$ is the direct sum of all different indecomposable summands of $M$ with support contained in the set $\{(j, l)$, for all $1 \leq j \leq$ $\left.b, 1 \leq l \leq p_{j}\right\} \cup\{\omega\}$,

- $M_{j}=I_{\left(j, e_{j}-1\right)}$ where $e_{j}=\min \{i / 1 \leq i \leq \omega$, such that there exists a relation in the ideal $I$ ending at $(j, i)\}$, for all $j: b+1 \leq j \leq b+m+f+o$. In case $e_{j}=\omega$ then take $p_{j}$ instead of $e_{j}-1$.

Using the additivity of functors Hom and Ext we obtain:

$$
\begin{aligned}
& \operatorname{Hom}_{A_{0}}(M, M) \simeq \operatorname{Hom}_{A_{0}}\left(M_{b}, M_{b}\right) \oplus\left(\bigoplus_{j=b+1}^{B-a} \operatorname{Hom}_{A_{0}}\left(M_{b}, M_{j}\right)\right) \\
& \oplus \operatorname{Hom}_{A_{0}}\left(M_{b}, P_{\omega}\right)^{a} \oplus \bigoplus_{j=b+1}^{B-a}\left[\operatorname{Hom}_{A_{0}}\left(M_{j}, M_{b}\right)\right. \\
&\left.\oplus \operatorname{Hom}_{A_{0}}\left(M_{j}, \bigoplus_{j^{\prime}=b+1}^{B-a} M_{j^{\prime}}\right) \oplus \operatorname{Hom}_{A_{0}}\left(M_{j}, P_{\omega}\right)^{a}\right] \\
& \oplus k^{(d-a) a} \oplus k^{a^{2}},
\end{aligned}
$$


and, for $i \geq 1$,

$$
\begin{aligned}
\operatorname{Ext}_{A_{0}}^{i}(M, M) & \simeq \operatorname{Ext}_{A_{0}}^{i}\left(M_{b}, M_{b}\right) \oplus \operatorname{Ext}_{A_{0}}^{i}\left(M_{b}, P_{\omega}\right)^{a} \\
& \oplus \bigoplus_{j=b+1}^{B-a}\left(\operatorname{Ext}_{A_{0}}^{i}\left(M_{j}, M_{b}\right) \oplus \operatorname{Ext}_{A_{0}}^{i}\left(M_{j}, P_{\omega}\right)^{a}\right),
\end{aligned}
$$

since $P_{\omega}$ is projective and $M_{j}$ is injective, for all $b+1 \leq j \leq B-a$.

It follows directly from Remark 3.2 that for all $j, j^{\prime}$ such that $b+1 \leq$ $j, j^{\prime} \leq B-a$ we have

$$
\operatorname{Hom}_{A_{0}}\left(M_{b}, M_{j}\right)=0
$$

$\operatorname{Hom}_{A_{0}}\left(M_{j}, M_{j^{\prime}}\right)= \begin{cases}0 & \text { for all } j \neq j^{\prime} \\ k & \text { if } j=j^{\prime} \text { (since } M_{j} \text { is indecomposable). }\end{cases}$

Similarly, let $M_{b}=\bigoplus_{h=1}^{r} V_{h}$ with $V_{h}$ all the different indecomposable summands of $M$ with support contained in the set $\{(j, l): 1 \leq j \leq$ $b$ and $\left.1 \leq l \leq p_{j}\right\} \cup\{\omega\}$. Therefore

$$
\operatorname{Hom}_{A_{0}}\left(M_{b}, M_{b}\right)=k^{r}
$$

Observe that, if $b+m+f+1 \leq j \leq b+m+f+o$, then $M_{j}$ is a projective module. Therefore, for all $j$ such that $b+m+f+1 \leq j \leq$ $b+m+f+o$ we have

$$
\operatorname{Ext}_{A_{0}}^{i}\left(M_{j},-\right)=0 .
$$

Now, in order to compute the rest of these groups we will consider

a) a projective resolution of $M_{b}$, denoted by $\mathbf{P}_{M_{b}}$

b) a projective resolution of $M_{j}$, denoted by $\mathbf{P}_{M_{j}}$, for $b+1 \leq j \leq$ $b+m+f$.

a) $\mathbf{P}_{M_{b}}: 0 \rightarrow P_{\omega}^{b-(d-a)} \rightarrow \bigoplus_{j=1}^{b} P_{(j, 1)} \rightarrow M_{b} \rightarrow 0$

By applying the functor $\operatorname{Hom}_{A_{0}}\left(-, P_{\omega}\right)$ to this sequence and using the fact that $\operatorname{Hom}_{A_{0}}\left(P_{(j, 1)}, P_{\omega}\right)=P_{\omega}((j, 1))=0$ we get 


$$
\operatorname{Ext}_{A_{0}}^{i}\left(M_{b}, P_{\omega}\right)= \begin{cases}0 & \text { if } i=0 \\ k^{b+a-d} & \text { if } i=1 \\ 0 & \text { if } i \geq 2 .\end{cases}
$$

Analogously, by applying the functor $\operatorname{Hom}_{A_{0}}\left(-, M_{b}\right)$ to $\mathbf{P}_{M_{b}}$ and recalling that $\operatorname{Hom}_{A_{0}}\left(P_{(j, 1)}, M_{b}\right)=M_{b}((j, 1))=k$ for all $1 \leq j \leq b$ and $\operatorname{Hom}_{A_{0}}\left(P_{\omega}, M_{b}\right)=M_{b}(\omega)=k^{d-a}$, we obtain the long exact sequence

$$
\begin{gathered}
0 \rightarrow \operatorname{Hom}_{A_{0}}\left(M_{b}, M_{b}\right) \rightarrow k^{b} \rightarrow k^{(d-a)(b+a-d)} \rightarrow \\
\operatorname{Ext}_{A_{0}}^{1}\left(M_{b}, M_{b}\right) \rightarrow 0 .
\end{gathered}
$$

Therefore,

$$
\operatorname{Ext}_{A_{0}}^{i}\left(M_{b}, M_{b}\right)= \begin{cases}k^{r} & \text { if } i=0 \\ k^{r-b+(d-a)(b+a-d)} & \text { if } i=1, \\ 0 & \text { if } i \geq 2\end{cases}
$$

since (III) holds.

b) For all $b+1 \leq j \leq b+m+f$,

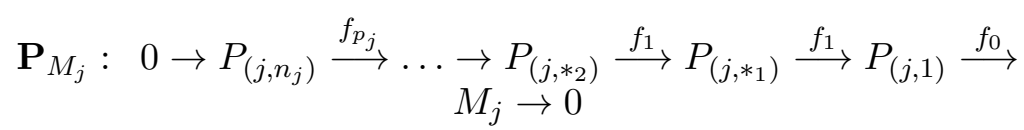

where:

- $n_{j}=*_{p_{j}}$ is the second component of the vertex where the first real overlapping ends;

- $p_{j}=\operatorname{pdim}\left(M_{j}\right)$.

It is easy to see that $p_{j}=\operatorname{pdim} M_{j}$ is the number of monomial relations that are involved in the first real overlapping [GHZ].

Let us denote $K_{(j, l)}=\operatorname{ker} f_{l}$ in $\mathbf{P}_{M_{j}}$ for $0 \leq l \leq p_{j}, K_{(j,-1)}=$ $M_{j}$ and $*_{0}=1$. Then, by applying the functor $\operatorname{Hom}_{A_{0}}\left(-, M_{b}\right)$ to the short exact sequence $0 \rightarrow K_{(j, l)} \rightarrow P_{\left(j, *_{l}\right)} \rightarrow K_{(j, l-1)} \rightarrow$ 0 we can infer that

$$
\operatorname{Ext}_{A_{0}}^{i}\left(M_{j}, M_{b}\right)=\operatorname{Hom}_{A_{0}}\left(K_{(j, i-1)}, M_{b}\right) \quad \forall i \geq 0,
$$

since $\operatorname{Hom}_{A_{0}}\left(P_{\left(j, *_{l}\right)}, M_{b}\right)=M_{b}\left(\left(j, *_{l}\right)\right)=0$.

We get by Remark 3.2 that

$$
\operatorname{Hom}_{A_{0}}\left(M_{j}, M_{b}\right)=0 \quad(V I I I) .
$$

In an analogous way, we can prove that 


$$
\begin{gathered}
\operatorname{Ext}_{A_{0}}^{i}\left(M_{j}, P_{\omega}\right)= \\
\left\{\begin{array}{ll}
0 & \text { if } i=0, \\
\operatorname{Hom}_{A_{0}}\left(K_{(j, i-1)}, P_{\omega}\right) & \text { if } i \geq 1 .
\end{array} \quad(I X),\right.
\end{gathered}
$$

(just replace the functor $\operatorname{Hom}_{A_{0}}\left(-, M_{b}\right)$ by the functor $\left.\operatorname{Hom}_{A_{0}}\left(-, P_{\omega}\right)\right)$.

Observe that $\omega \in \operatorname{supp} P_{\left(j, *_{l}\right)}$ implies $p_{j}-1 \leq l \leq p_{j}$.

Therefore

$$
\begin{gathered}
\operatorname{Hom}_{A_{0}}\left(K_{\left(j, *_{l}\right)}, M_{b}\right)=0=\operatorname{Hom}_{A_{0}}\left(K_{\left(j, *_{l}\right)}, P_{\omega}\right), \forall l< \\
p_{j}-1, \quad(X)
\end{gathered}
$$

because supp $K_{\left(j, *_{l}\right)} \subset \operatorname{supp} P_{\left(j, *_{l}\right)}$.

On the other hand, $\operatorname{Hom}_{A_{0}}\left(K_{\left(j, p_{j}\right)}, M_{b}\right) \neq 0$ and $\operatorname{Hom}_{A_{0}}\left(K_{\left(j, p_{j}\right)}, P_{\omega}\right) \neq 0$ if and only if $\operatorname{top} K_{\left(j, p_{j}\right)}=K_{\left(j, p_{j}\right)}=$ $S_{\omega}$. Then we conclude that both $\operatorname{Hom}_{A_{0}}\left(K_{\left(j, p_{j}\right)}, M_{b}\right)$ and $\operatorname{Hom}_{A_{0}}\left(K_{\left(j, p_{j}\right)}, P_{\omega}\right)$ are non zero if and only if $b+m+1 \leq$ $j \leq b+m+f$ and the real overlapping associated is full.

In this case

$\operatorname{Hom}_{A_{0}}\left(K_{\left(j, p_{j}\right)}, M_{b}\right)=k^{(d-a)}$ and $\operatorname{Hom}_{A_{0}}\left(K_{\left(j, p_{j}\right)}, P_{\omega}\right)=k,(X I)$.

Hence, the statement follows from (I)-(XI).

Now, our main result follows from Corollary 2.3, Theorem 2.4 and Lemma 3.3.

Theorem 3.4. Let $A$ be a toupie algebra. Then

$$
H^{i}(A)= \begin{cases}k & \text { if } i=0 \\ k^{r+m+f+o+d a-1} & \text { if } i=1, \\ k^{r-b+(b+a-d+f(1)) d} & \text { if } i=2, \\ k^{f(i-1) d} & \text { if } i \geq 3\end{cases}
$$

Remark 3.5. Another technique to obtain the Hochschild cohomology groups of toupie algebras could be the long exact sequence of vector spaces given by $[\mathrm{MiP}]$ related to triangular matrix algebras. In this case, it will be necessary to find convenient algebras and appropriate parameters. 
Example 3.6. Let $A=k Q / I$ be a canonical algebra in the sense of $[\mathrm{R}]$ (a particular case of toupie algebra). In this case $m=f=o=$ $a=0$ and this implies that $H^{0}(A)=k, H^{1}(A)=0, H^{2}(A)=b-3$ and $H^{i}(A)=0$, for all $i \geq 3$.

We remark that Happel proved in $[\mathrm{H} 2]$ that: $H^{0}(A)=k$ and $H^{i}(A)=$ 0 , for all $i \geq 3$.

Example 3.7. Let $A=k Q / J^{N}$ be a truncated toupie algebra. In this case $b=r=d-a, m=0$ and $o=0$. Following $[\mathrm{L}]$ and bearing in mind that a toupie quiver has no loops and for each $i \geq 0$ the $j$-extreme paths are exactly those parallel to paths of length $N i$ we get

- $H^{0}(A)=Z(A)$

- $\operatorname{dim}_{\mathrm{k}} H^{1}(A)=1-\sharp Q_{0}+\sum_{\alpha \in Q_{1}} \operatorname{dim}_{\mathrm{k}} o(\alpha) A t(\alpha)$;

- for all $i \geq 1$,

$\operatorname{dim}_{\mathrm{k}} H^{2 i}(A)=\sum_{j=1}^{N-1} \sharp\{$ paths of length $j$ parallel to paths of length $(N i)\}$.

and

$\operatorname{dim}_{\mathrm{k}} H^{2 i+1}(A)=\sum_{j=1}^{N-1} \sharp\{$ paths of length $j$ parallel to paths of length $(N i+1)\}$

Therefore

- $\operatorname{dim}_{\mathrm{k}} H^{1}(A)=1-\sharp Q_{0}+\sum_{\alpha \in Q_{1}} \operatorname{dim}_{\mathrm{k}} o(\alpha) A t(\alpha)=1-\sharp Q_{0}+\left\{\sharp Q_{0}\right.$

$-2+r+f+($ d. $a)\}$

- for all $i \geq 1$, 


$$
\begin{aligned}
\operatorname{dim}_{\mathrm{k}} H^{2 i}(A) & =\sum_{j=1}^{N-1} \sharp\{\text { paths of length } j \text { parallel to paths of length }(N i)\} \\
& =\sharp\{\text { paths of length } 1 \text { parallel to paths of length }(N i)\} \\
& +\sum_{j=2}^{N-1} \sharp\{\text { paths of length } j \text { parallel to paths of length }(N i)\} \\
& =a \cdot \sharp\{\text { paths of length }(N i)\}+b . \sharp\{\text { paths of length }(N i)\} \\
& =d \cdot \sharp\{\text { paths of length }(N i)\} \\
& =d . f(2 i-1) .
\end{aligned}
$$

where the last equality holds by computing the real overlapping associated to paths of length $\mathrm{Ni}$.

Similar arguments give $\operatorname{dim}_{\mathrm{k}} H^{2 i+1}(A)=\operatorname{d.f}(2 i-1)$.

Corollary 3.8. The cup product of the Hochschild cohomology ring of a toupie algebra is trivial.

Proof. By Theorem 2.5 we have that the graded homomorphism associated to the Happel long exact sequence $\delta^{*}: \operatorname{Ext}_{A_{0}}^{*}[M, M] \rightarrow$ $H^{*}(A)[-1]$ has image in the annihilator of $\coprod_{n \geq 1} H^{n}(A)$. Since $H^{i}\left(A_{0}\right)=$ 0 , for all $i \geq 1$, we have that $\delta^{i}$ is an epimorphism, and we get the result.

\section{Applications}

As we mentioned in the introduction, tight links exist between the vanishing of the low dimensional Hochschild cohomology groups, the simple connectedness and the rigidity of an algebra. We will refer to these subjects in the following two corollaries.

We start by defining the fundamental group of a bound quiver. Let $(Q, I)$ be a connected bound quiver. A relation $\rho=\sum_{i=1}^{m} \lambda_{i} w_{i}$ in $I(x, y)$ is called minimal if $m \geq 2$ and for every non-empty proper subset $J \subset\{1,2, \ldots, m\}$ we have that $\sum_{j \in J} \lambda_{j} w_{j} \notin I(x, y)$. 
For an arrow $\alpha \in Q_{1}$, we denote by $\alpha^{-1}$ its formal inverse. A walk from $x$ to $y$ in $Q$ is a formal composition $\alpha_{1}^{\epsilon_{1}} \alpha_{2}^{\epsilon_{2}} \ldots \alpha_{t}^{\epsilon_{t}}$ (where $\alpha_{i} \in Q_{1}$, $\epsilon_{i}={ }_{-}^{+} 1$ for $\left.1 \leq i \leq t\right)$ starting at $x$ and ending at $y$.

We define the homotopy relation $\sim$ to be the smallest equivalence relation on the set of all walks in $Q$ such that:

a) If $\alpha: x \rightarrow y$ is an arrow then $\alpha^{-1} \alpha \sim e_{x}$ and $\alpha \alpha^{-1} \sim e_{y}$.

b) If $\rho=\sum_{i=1}^{m} \lambda_{i} w_{i}$ is a minimal relation then $w_{i} \sim w_{j}$ for all $1 \leq i, j \leq m$.

c) If $u \sim v$ then $w u w^{\prime} \sim w v w^{\prime}$ whenever these compositions are defined.

We denote by $[u]$ the equivalence class of a walk $u$.

Let $x_{0} \in Q_{0}$ be arbitrary, the set $\pi_{1}\left(Q, I, x_{0}\right)$ of equivalence classes of all the closed walks starting and ending at $x_{0}$ has a group structure defined by the operation $[u][v]=[u v]$. Clearly the group $\pi_{1}\left(Q, I, x_{0}\right)$ does not depend on the choice of the base point $x_{0}$. We denote it simply by $\pi_{1}(Q, I)$ and call it the fundamental group of $(Q, I)$ (see $[\mathrm{G}],[\mathrm{MP}])$.

It follows from the definition that the fundamental group $\pi_{1}(Q, I)$ depends essentially on $I$, thus it is not an invariant of $A$, see, for instance $[\mathrm{AP}]$.

Example 4.1. Consider the following toupie quiver $Q$ and the ideal $I=<\delta_{2} \delta_{1}, \delta_{3} \delta_{2}, \alpha_{3} \alpha_{2} \alpha_{1}+\beta_{3} \beta_{2} \beta_{1}+\gamma_{3} \gamma_{2} \gamma_{1}>$.

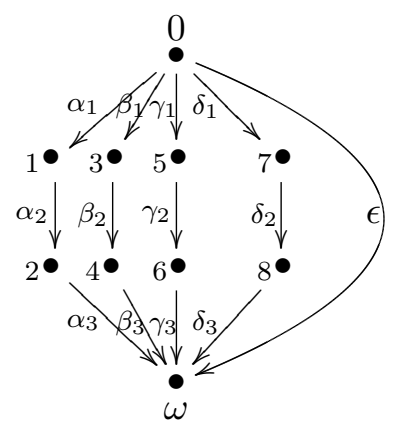

We compute the fundamental group of this presentation $(Q, I)$ using the algorithm given by [Re]: 

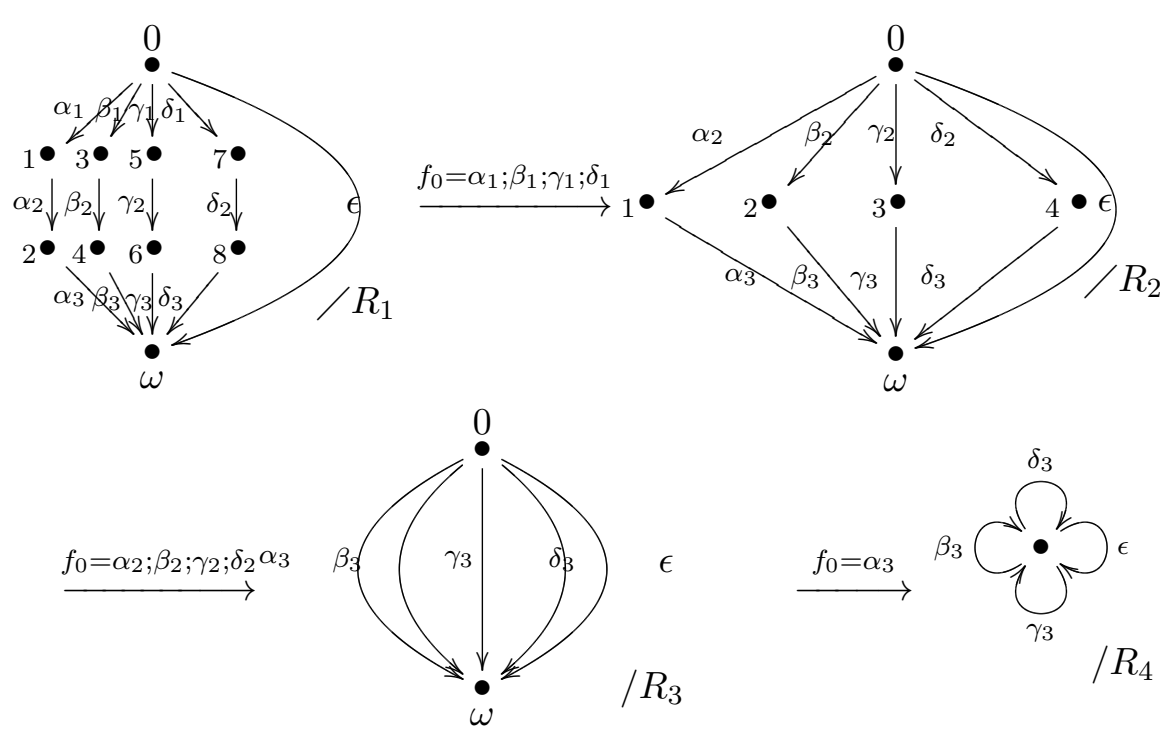

with: $\left\{\begin{array}{l}R_{1}=<\alpha_{3} \alpha_{2} \alpha_{1} \sim \beta_{3} \beta_{2} \beta_{1}, \beta_{3} \beta_{2} \beta_{1} \sim \gamma_{3} \gamma_{2} \gamma_{1}> \\ R_{2}=<\alpha_{3} \alpha_{2} \sim \beta_{3} \beta_{2}, \beta_{3} \beta_{2} \sim \gamma_{3} \gamma_{2}> \\ R_{3}=<\alpha_{3} \sim \beta_{3}, \beta_{3} \sim \gamma_{3}> \\ R_{4}=<s\left(\beta_{3}\right) \sim \beta_{3}, \beta_{3} \sim \gamma_{3}>.\end{array}\right.$

Then, after simplification of arrows $\beta_{3}$ and $\gamma_{3}$ we get that $\pi_{1}(Q, I)$ is the free group generated by two elements.

A connected triangular algebra $A$ is called simply connected if for any presentation $\left(Q_{A}, I\right)$ of $A$, the fundamental group $\pi_{1}\left(Q_{A}, I\right)$ is trivial $([\mathrm{AP}])$. In general, it may be complicated to verify when an algebra $A$ is simply connected since the fundamental groups of two presentations of $A$ can be completety different (see $[\mathrm{BC}])$. In $[\mathrm{BM}]$, Bardzell and Marcos proved that if the algebra is constricted (that is, $\left.\forall \alpha \in Q_{1} \operatorname{dim}_{\mathrm{k}} e_{t(\alpha)} A e_{s(\alpha)}=1\right)$ then the fundamental group is independent of the presentation.

The next corollary, known for representation finite algebras [BL], strictly weakly shod algebras ( $[\mathrm{AL}],[\mathrm{Le}])$ and laura algebras [ABLe], gives a direct way to determinate the simple connectedness of the toupie algebras. 
Corollary 4.2. Let $A$ be a toupie algebra.

Then $A$ is simply connected if and only if $H^{1}(A)=0$.

Proof. It is not difficult to see, as in Example 4.1, that $\pi_{1}(Q, I)$ is the free group generated by $r+m+f+o+a-1$ elements ([B, Re]). If $H^{1}(A)=0$ then, by Theorem 3.4, $r+m+f+o+(d-a) \cdot a+a^{2}=1$. Since $r \geq 0, m \geq 0, f \geq 0, o \geq 0, a \geq 0$ and $(d-a) . a \geq 0$ then

(I) $r+m+f+o=1$ and $a^{2}=0$ or,

(II) $a=1, d=1$ and $r=m=f=o=0$.

Therefore, $\pi_{1}(Q, I)=0$. It is clear that in both cases, the toupie algebra is constricted, so $A$ is simply connected.

Conversely, if $A$ is simply connected then $\pi_{1}(Q, I)=0$ for all presentation $(Q, I)$ of $A$. So, $r+m+f+o+a-1=0$ and therefore the only possibilities are that I) or II) hold. Then, by Theorem 3.4, $H^{1}(A)=0$.

Remark 4.3. Notice that if $H^{1}(A)=0$ then we can describe the presentation of the algebra toupie (recall that all parameters are non negative integer numbers).

Corollary 4.4. Let $A=k Q / I$ be a toupie algebra.

Then $H^{2}(A)=0$ if and only if af $(1)+b=0$.

Proof. Since the converse is trivial, assume that $H^{2}=0$. Then, by Theorem 3.4, $r-b+(b+a-d+f(1)) d=0$.

The number $r-b+(b+a-d) d$ corresponds to the second Hochschild cohomology dimension for the toupie algebra $A^{\prime}=k Q^{\prime} / I^{\prime}$ where $Q^{\prime}$ is the maximal full subquiver of $Q$ with $f_{Q^{\prime}}(1)=0$ and $I^{\prime}=I \cap k Q^{\prime}$. Therefore $0=r-b+(b+a-d+f(1)) d \geq r-b+(b+a-d) d \geq 0$ and this implies $r-b+(b+a-d) d=0$. Now $r-b+(b+a-d) \bar{d} \geq$ $r-b+(b+a-d)(d-a)$ and the last expression corresponds to the second Hochschild cohomology dimension for the toupie algebra $A^{\prime \prime}=k Q^{\prime \prime} / I^{\prime \prime}$ where $Q^{\prime \prime}$ is the maximal full subquiver of $Q$ with $a_{Q^{\prime \prime}}=0$ (that is, there is no arrows from 0 to $\omega)$. Therefore $r-b+(b+a-d)(d-a)=0$, hence $d-a=0$ since $r \leq d-a \leq b$ (in particular $b=r=0$ ).

An algebra $A$ is said to be rigid if any one-parameter deformation is isomorphic to the trivial one, see [G]. It is known that if $H^{2}(A)=0$ 
then $A$ is rigid. Moreover, if $H^{3}(A)=0$, the converse is also true. Then, we have the following straightforward consequence of Theorem 3.4 and Corollary 4.4 .

Corollary 4.5. Let $A=k Q / I$ be a toupie algebra.

(1) If $a f(1)+b=0$ then $A$ is rigid.

(2) If $a=0$ then: $A$ is rigid if and only if $b=0$.

We finish the paper providing applications of the results obtained. More precisely, using Theorem 3.4 and Theorem 1 of [BDS] we can compute the Hochschild cohomology groups of a particular family of algebras which arise from gluing together several toupie algebras sinks with sinks, or sources.

Example 4.6. Let $C$ be the algebra with associated underlying graph:

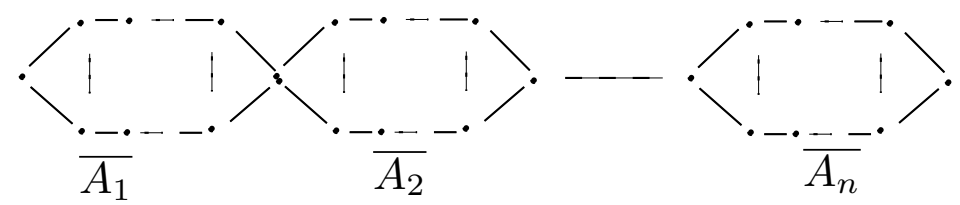

FiguRE 1. Chain algebra

where $n \geq 2$ and, for all $j \geq 1, \overline{A_{j}}$ denotes the underlying graph corresponding to a toupie algebra $A_{j}$.

If $C$ verifies that

$$
\operatorname{dim}_{\mathrm{k}} e_{x} C e_{y} \neq 0 \Rightarrow x, y \in Q_{0}\left(A_{j}\right)
$$

then

$$
H^{i}(C)=\oplus_{j=1}^{n} H^{i}\left(A_{j}\right) .
$$

In fact, using recurrence over $n \geq 2$, this is a consequence of the result given by Bustamante, Dionne and Smith (see Theorem 1 of [BDS]) concerning oriented pullbacks of algebras.

Moreover, using the terminology of [BDS], $C$ is a simple articulated algebra and using Corollary 3.3 .3 of [BDS] and Corollary 3.5 we obtain that $C$ is simply connected if and only if $H^{1}(C)=0$. 
Example 4.7. Using the same arguments and notations of the previous example, it is easy to compute all the Hochschild cohomology groups of the following algebras $F$ and $B$ with underlying graph:

I) $F$ :

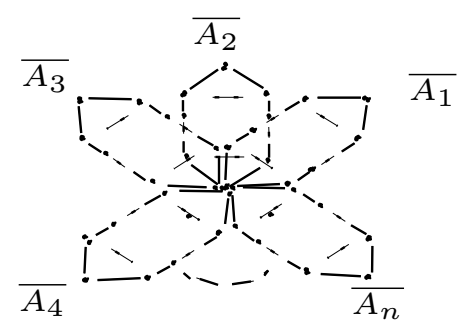

FiguRE 2. Flower algebra

where $n \geq 2$ and, for all $j \geq 1, \overline{A_{j}}$ denotes the underlying graph corresponding to a toupie algebra $A_{j}$.

If $F$ verifies that

$$
\operatorname{dim}_{\mathrm{k}} e_{x} F e_{y} \neq 0 \Rightarrow x, y \in Q_{0}\left(A_{j}\right)
$$

then

$$
H^{i}(F)=\oplus_{j=1}^{n} H^{i}\left(A_{j}\right) \text { for all } i \geq 1
$$

and

$$
F \text { is simply connected if and only if } H^{1}(F)=0 \text {. }
$$

II) $B$ :

where $n \geq 2$ and, for all $j \geq 1, \overline{A_{j}}$ denotes the underlying graph corresponding to a toupie algebra $A_{j}$.

If $B$ has a sink and a source not belonging to the same toupie (in particular, not all algebras $A_{j}$ have the same orientation) and verifies that $\operatorname{dim}_{\mathrm{k}} e_{x} B e_{y} \neq 0 \Rightarrow x, y \in Q_{0}\left(A_{j}\right)$ then

$$
H^{i}(B)=\oplus_{j=1}^{n} H^{i}\left(A_{j}\right) \text { for all } i \geq 2
$$




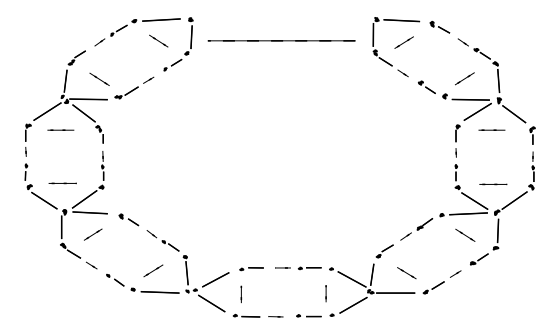

Figure 3. Bracelet algebra

and

$$
\operatorname{dim}_{\mathrm{k}} H^{1}(B)=\oplus_{j=1}^{n} \operatorname{dim}_{\mathrm{k}} H^{1}\left(A_{j}\right)-1
$$

\section{References}

[AL] I. Assem and M. Lanzilotta, The simple connectedness of a tame weakly shod algebra, Comm. Algebra 32, 9 (2004), 3685-3701.

[AP] I. Assem and J. A. de la Peña, The fundamental groups of a triangular algebra, Comm. Algebra 24, 1 (1996), 187-208.

[ABLe] I. Assem, J. C. Bustamente and P. Le Meur, Coverings of Laura algebras: the Standard Case, hal-00310452, version 1 - August 8, 2008.

[ARS] M. Auslander, I. Reiten and S. Smalø, Representation theory of artin algebras, Cambridge Studies in Advanced Mathematics 36, 1995.

[BLM] M.J. Bardzell, A. C. Locateli and E. Marcos, On the Hochschild cohomology groups of truncated cycle algebras, Comm. Algebra 28, 3 (2000), 1615-1639.

[BM] M.J. Bardzell and E. Marcos, $H^{1}(\Lambda)$ and presentations of finite dimensional algebras, Lecture Notes in Pure and Appl. Math. 224, 31-38, Dekker, N.Y, 2001.

[BL] R. O. Buchweitz and S. Liu, Hochschild cohomology and representationfinite algebras, Proc. London Math. Soc. (3) 88 (2004), $\mathrm{n}^{\mathrm{o}}$ 2, 355-380.

[B] J. C. Bustamante. On the fundamental group of a shurian algebra, Comm. Algebra 30, 11 (2002), 5305-5327.

[BC] J. C. Bustamante and D. Castonguay Fundamental groups and presentations of algebras, archiv:math/0405127, preprint.

[BDS] J. C. Bustamante, J. Dionne and D. Smith, (Co)homologies theory for oriented algebras, Comm. Algebra 37 (2009), n ${ }^{\circ}$ 5, 1516-1544.

[C1] C. Cibils, On the Hochschild cohomology of finite dimensional algebras, Comm. Algebra 16, 3 (1988), 645-649.

[C2] C. Cibils, Hochschild cohomology algebra of radical square zero algebras, Algebras and Modules II, CMS Conf. Proc. 24, 93-101, Providence, RI, 1998, Amer. Math. Soc. 
[C3] C. Cibils, Tensor Hochschild homology and cohomology, Interactions between ring theory and representations of algebras, Lecture Notes in Pure and Appl. Math. 210, 35-51, Dekker, NY, 2000.

[CDHL] D. Castonguay, J. Dionne, F. Huard, M. Lanzilotta, Toupie algebras, preprint (2008).

[GR1] M.A. Gatica and M.J. Redondo, Hochschild cohomology and fundamental groups of incidence algebras, Comm. Algebra 29, 5 (2001), 2269-2283.

[GR2] M.A. Gatica and M.J. Redondo, Hochschild cohomology of incidence algebras as one point extensions, Linear Algebra Appl. 365 (2003), 169 181 .

[GRey] M.A. Gatica and A.A. Rey, Computing the Hochschild cohomology groups of special kinds of incidence algebras, Comm. Algebra 34, 6 (2006), 2039-2056

[G] M. Gerstenhaber, On the deformations of rings and algebras, Ann. of Math. (2) 79 (1964), 59-103.

[Gr] E. L. Green Graphs with relations, coverings and group-graded algebras, Trans. Amer. Math. Soc. 279, 1 (1983), 279-310.

[GHZ] E. L. Green, D. Happel, D. Zacharia, Projective resolutions over artin algebras with zero relations, Ill. J. Math., Vol. 29, 1 (1985), 180-190.

[GMS] E. L. Green, E. N. Marcos, N. Snashall, The Hochschild cohomology ring of a one-point extension, Comm. Algebra, Vol. 31, 1 (2003), 357-379.

[H1] D. Happel, Hochschild cohomology of finite-dimensional algebras, Séminaire d'Algèbre Paul Dubreil et Marie Paul Malliavin, 39ème Année (Paris, 1987/1988), 108-126, Lecture Notes in Math. 1404, Springer, Berlin (1989).

[H2] D. Happel, Hochschild cohomology of piecewise hereditary algebras, Coll. Math. 78, 2, (1998), 261-266.

[Ho] G. Hochschild, On the cohomology groups of an associative algebra, Ann. of Math. 46, 2 (1945), 58-67.

[Le] P. Le Meur, Galois coverings of weakly shod algebras, to appear, 2008.

[L] A.C. Locateli, Hochschild cohomology of truncated quiver algebras, Comm. Algebra 27, 2 (1999), 645-664.

[MP] R. Martínez Villa and J. A. de la Peña, The universal cover of a quiver with relations, J. Pure Appl. Algebra 30, 3 (1983), 277-292.

[MiP] S. Michelena and M. I. Platzeck, Hochschild cohomolgy of triangular matrix algebras, J. Algebra 233, 2 (2000), 502-525.

[Re] E. Reynaud, Algebraic fundamental group and simplicial complexes, J. Pure Appl. Algebra 177, 2 (2003), 203-214.

[R] C. M. Ringel, Tame algebras and integral quadratic forms, Lecture Notes in Mathematics, 1099, Springer-Verlag, Berlin, 1984. 